\title{
CARDIAC HYPERTROPHY IN COALWORKERS' PNEUMOCONIOSIS
}

\author{
BY \\ W. R. L. JAMES and ARTHUR J. THOMAS \\ From the Department of Pathology and Bacteriology, Welsh National School of Medicine, Cardiff, and \\ Llandough Hospital, the United Cardiff Hospitals
}

(RECEIVED FOR PUBLICATION MAY 22, 1955)

\begin{abstract}
Pneumoconiosis of coalworkers is a pulmonary disease which can produce a striking effect on the heart (Thomas, 1948; Lavenne, 1951; Wells, 1954a). Many coalworkers in the southern part of Wales develop the pulmonary lesions. In some there are numerous foci of accumulated dust scattered more or less uniformly throughout each lung ; the foci are discrete, usually less than $7 \mathrm{~mm}$. in diameter, and contain little or no collagenous fibrous tissue. This condition is called simple pneumoconiosis. There may be areas of focal emphysema in and around the dust foci (Heppleston, 1953). The lungs of other miners show in addition to simple pneumoconiosis one or more dustpigmented fibrous lesions. Initially, these fibrous areas are less than $1 \mathrm{~cm}$. across but slowly enlarge and in the later stages more than half of each lung may be destroyed by the fibrosis. This condition is referred to as progressive massive fibrosis or massive pneumoconiosis. It is often complicated by bullous or diffuse emphysema (Fletcher and Gough, 1950).
\end{abstract}

The presence of pulmonary heart disease becomes obvious when right heart failure appears but this is a late and often terminal event, and it was of interest to assess earlier stages of pulmonary heart disease in relation to the extent of the pneumoconiosis. This work therefore compares the right ventricle studied at necropsy with the pulmonary disease, and relates the electrocardiogram to the necropsy findings in some cases.

The electrocardiographic signs of pulmonary heart disease used in this study have been the vertical heart pattern or right axis deviation with well developed pointed $\mathbf{P}$ in leads II and III, T inversion in lead III or downward inclination of the ST segment in II and III, a qR pattern in VR, a deep $S$ wave in $V 6$ and $R$ greater than $S$ in V1, or M-shaped QRS complex in V1. The unusual standard lead pattern of prominent $S_{I}, S_{I I}$, and $S_{\text {III }}$ has also been accepted in conjunction with the other signs. Not all the above signs were present in every case, but most of them had to be present before the electrocardiogram was regarded as indicative of pulmonary heart disease.

Routine necropsies have been used for part of this work and in these a thickness of $5 \mathrm{~mm}$. or more of the right ventricular wall has indicated hypertrophy. A selected number of hearts have been dissected and the ventricles weighed using the method of Herrmann and Wilson (1922). By this method the normal range of the ratio of left ventricle to right ventricle (LV/RV ratio) was 1.46 to $2 \cdot 14$. Thus ratios below 1.46 indicate right ventricular hypertrophy but it was found in practice that ratios below 1.00 were comparable to a thickness of $5 \mathrm{~mm}$. or more, and this group is referred to as marked right ventricular hypertrophy. The intermediate group with an LV/RV ratio lying between 1.46 and 1 and not identified by thickness of the wall is referred to as early right ventricular hypertrophy.

All the material used in this study was of necessity selective but as the object was to study the relationship between cardiac hypertrophy and pneumoconiosis rather than the absolute incidence of the phenomenon the material was acceptable.

\section{Results}

The overall pattern is shown by a scrutiny of the protocols of the necropsies made on 1,000 coalworkers during the years 1947-50 at the Institute of Pathology of the Welsh National School of Medicine. These were cases coming to necropsy because of a history of exposure to coal dust but the causes of death were various. Of the 1,000 cases, 546 had simple pneumoconiosis and 454 had massive fibrosis. The latter was diagnosed if either lung contained a dust-pigmented fibrous lesion more than $3 \mathrm{~cm}$. across.

Of the 546 cases with simple pneumoconiosis, only eight were considered at necropsy to have died 
of pulmonary heart disease due to the simple dust disease alone. This opinion was based on a clinical history of congestive heart failure and naked-eye pathological evidence of severe simple pneumoconiosis with right ventricular hypertrophy and a lack of any other cause for the right cardiac enlargement. In a further seven cases with right ventricular hypertrophy death was not due to the simple pneumoconiosis but the latter appeared to be the only cause for the right ventricular change. Thus, on the basis of gross post-mortem appearances the series contained 15 cases in which the simple dust disease alone had affected the right heart. The lungs in these cases were subsequently studied microscopically and by the use of whole lung sections (Gough and Wentworth, 1949), and this investigation showed evidence in each case of chronic inflammation of the smaller bronchi and of slight degrees of diffuse emphysema. It was not possible to prove that the co-existing chronic bronchiolitis or emphysema was due to the simple pneumoconiosis. On the contrary, Gough (1952) has drawn attention to a centrilobular focal type of emphysema with bronchiolitis in lungs free of dust. Moreover, Leopold (1955) has shown that right ventricular hypertrophy may result from chronic bronchiolitis. It is, therefore, possible that in the 15 cases of simple pneumoconiosis described the right ventricular hypertrophy was due partly to bronchiolitis or emphysema. We had to conclude that simple pneumoconiosis alone occasionally caused right heart hypertrophy, but would suggest that chronic bronchiolitis also played a part.

The 454 cases of massive fibrosis included 412 with other co-existing lung lesions of various degrees. The common co-existing lesions were generalized emphysema, focal emphysema, chronic bronchiolitis, and pulmonary tuberculosis. In the 43 remaining cases the lungs showed massive pneumoconiosis only. The extent of the emphysema was judged by naked-eye examination of the fresh lungs and the whole lung sections. It was deemed to be insignificant if it was similar in extent to that seen in the lungs of individuals who have not had symptoms of emphysema. For example, the small emphysematous areas shown in Fig. 1 were deemed to be insignificant. The 43 cases were classified according to the size of the massive dust lesion. When the lungs contained more than one lesion the classification was based on the maximum diameter of the largest one. In nine the diameter was less than $5 \mathrm{~cm}$., in 15 it was between 5 and $10 \mathrm{~cm}$., and in 19 it was over $10 \mathrm{~cm}$. The cases were also classified according to the presence or absence of right ventricular hypertrophy. The latter was deemed to

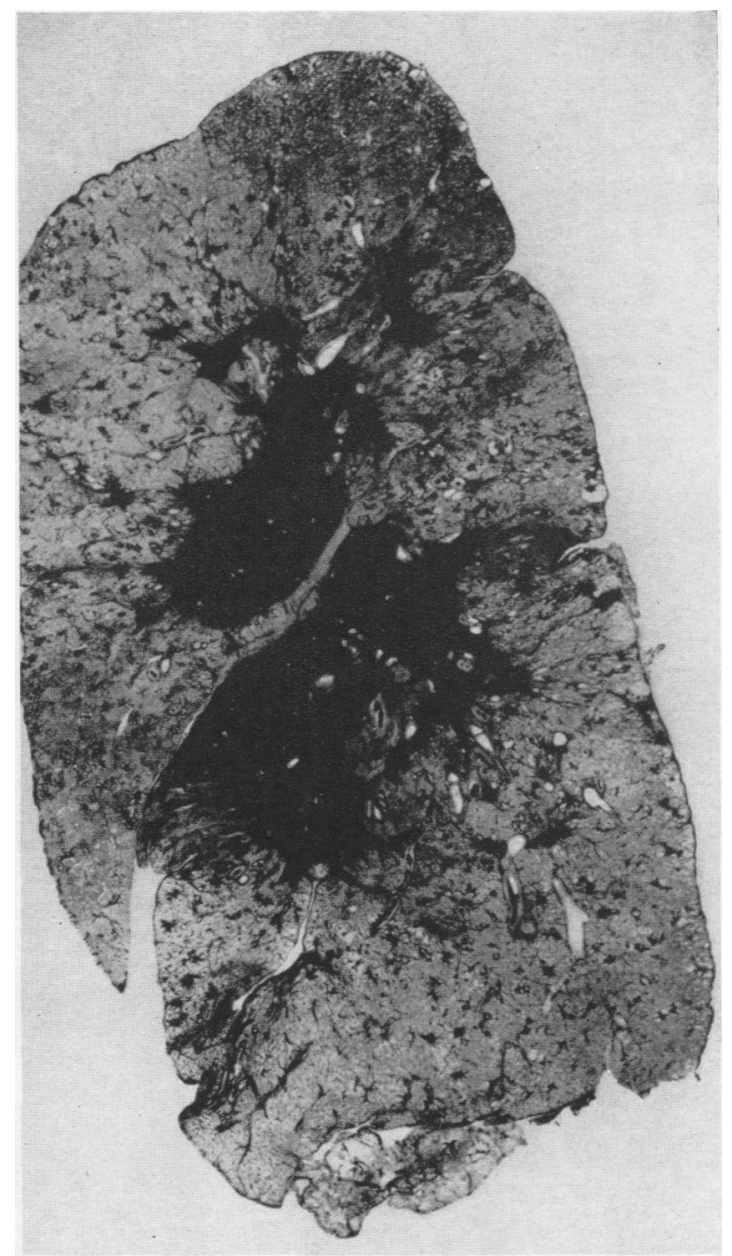

Fig. 1.-Lung section (about half natural size) from a case in which progressive massive fibrosis with minimal emphysema had produced marked right ventricular hypertrophy.

be present if the maximum right ventricular thickness was over $5 \mathrm{~mm}$. The 43 cases were then analysed to determine whether any relationship existed between the size of the lesions and the incidence of right ventricular hypertrophy. The analysis is displayed in Table 1.

TABLE 1

INCIDENCE OF RIGHT VENTRICULAR HYPERTROPHY ACCORDING TO SIZE OF LESION

\begin{tabular}{|c|c|c|c|c|}
\hline & $\begin{array}{l}\text { Less than } \\
5 \mathrm{~cm} \text {. }\end{array}$ & $5-10 \mathrm{~cm}$ & $\begin{array}{c}\text { Over } \\
10 \mathrm{~cm}\end{array}$ & Total \\
\hline $\begin{array}{l}\text { Right ventricular } \\
\text { hypertrophy } \\
\text { Right ventricular } \\
\text { hypertrophy absent }\end{array}$ & $\begin{array}{l}1 \\
8\end{array}$ & $\begin{array}{r}13 \\
2\end{array}$ & $\begin{array}{r}17 \\
2\end{array}$ & $\begin{array}{l}31 \\
12\end{array}$ \\
\hline Totals & 9 & 15 & 19 & 43 \\
\hline
\end{tabular}


On studying the ages of these cases it was noted that the mean age of those with small lesions was 52 years, with moderately sized lesions 65 years, and with large lesions 60 years. It was possible, therefore, that increased age contributed somewhat to increased incidence of right ventricular hypertrophy, but the figures also show that the incidence of right ventricular hypertrophy increased with increasing size of the dust lesion in the cases of massive pneumoconiosis and minimal emphysema.

In simple pneumoconiosis right ventricular hypertrophy was an uncommon finding, 15 in 546 cases, and in these there was a degree of chronic bronchiolitis whose importance needs further investigation. Right ventricular hypertrophy was common in massive fibrosis. Cases of pure massive fibrosis were not common, 43 in 454 cases, but of the 43 there were 31 with marked right ventricular hypertrophy, suggesting a relationship between massive fibrosis alone and right ventricular hypertrophy.

Eighty hearts from necropsies on coalworkers were dissected and the ventricles weighed (Table 2).

TABLE 2

ANALYSIS OF VENTRICULAR WEIGHTS OF 80 PNEUMOCONIOTIC HEARTS

\begin{tabular}{l|c|c|c|c}
\hline $\begin{array}{c}\text { Stage of } \\
\text { Disease }\end{array}$ & $\begin{array}{c}\text { Marked Right } \\
\text { Ventricular } \\
\text { Hypertrophy } \\
\text { (LV/RV ratio } \\
\text { less than 1) }\end{array}$ & $\begin{array}{c}\text { Early Right } \\
\text { Ventricular } \\
\text { Hypertrophy } \\
\text { (LV/RV ratio } \\
1.00 \text { to 1.46) }\end{array}$ & $\begin{array}{c}\text { Normal } \\
\text { LV/RV } \\
\text { Ratio } \\
\text { above } \\
1.46\end{array}$ & Totals \\
\hline $\begin{array}{c}\text { Progressive } \\
\text { massive } \\
\text { fibrosis } \\
\begin{array}{c}\text { Simple pneumo- } \\
\text { coniosis }\end{array}\end{array}$ & 32 & 19 & 5 & 56 \\
\hline
\end{tabular}

Fifty-six cases had progressive massive fibrosis. Thirty-two of these had marked hypertrophy with an $L V / R V$ ratio below 1,19 were cases of early right hypertrophy with an $L V / R V$ ratio between 1 and 1.46 , and five were normal. Of the 19 patients with early right hypertrophy, eight died of conditions not directly related to pneumoconiosis and 11 died of heart failure due to pneumoconiosis and emphysema. It was apparent that massive fibrosis was associated with some degree of right ventricular hypertrophy in all but five of the 56 cases, but in the 19 with early right ventricular hypertrophy clinical evidence of pulmonary heart disease would be difficult to find before the onset of right heart failure.

There were 24 cases of simple pneumoconiosis four of which had a ratio below 1 indicating marked right ventricular hypertrophy. In three of these there was severe bullous emphysema and the fourth had had anaemia for a long period.
Early right ventricular hypertrophy (LV/RV ratio between 1.46 and 1) was present in 11 cases. In five of these emphysema of considerable degree was present, two had pulmonary tuberculosis, and another had severe chronic bronchitis. These factors may have been contributory causes of the early right ventricular hypertrophy. The three remaining cases of simple pneumoconiosis appeared to have this as the only explanation of the early right ventricular hypertrophy.

It was of interest that in five of the cases of early right ventricular hypertrophy with electrocardiograms available not one electrocardiogram had the signs of right ventricular hypertrophy but all five had pulmonary $\mathbf{P}$ waves and all but one had a vertical heart pattern.

This analysis suggested that the right ventricular hypertrophy found in cases of massive fibrosis was frequently of a much greater degree than that found in simple pneumoconiosis. In a previous work (Thomas, 1951) it was suggested that the LV/RV ratio had to fall below 1, i.e., marked right ventricular hypertrophy had to be present, before electrocardiographic signs of it were seen. This would mean that right ventricular hypertrophy would be detected clinically with greater frequency in massive fibrosis than in simple pneumoconiosis.

In 56 clinical cases which came to necropsy the association of the electrocardiographic signs with the extent of the pneumoconiosis was studied (Table 3). The causes of death were various and not always related to the pneumoconiosis.

TABLE 3

RESULTS OF NECROPSY IN CLINICAL CASES OF PNEUMOCONIOSIS

\begin{tabular}{|c|c|c|c|c|}
\hline Stage of Disease & & $\begin{array}{l}\text { No. of } \\
\text { Cases }\end{array}$ & $\begin{array}{c}\text { Marked Right } \\
\text { Ventricular } \\
\text { Hypertrophy }\end{array}$ & \begin{tabular}{|} 
Pulmonary \\
Heart \\
Disease \\
on E.C.G.
\end{tabular} \\
\hline $\begin{array}{l}\text { Advanced massive fibrosis } \\
\text { Early massive fibrosis } \\
\text { Simple pneumoconiosis }\end{array}$ & $\begin{array}{l}\cdots \\
\cdots\end{array}$ & $\begin{array}{r}32 \\
17 \\
7\end{array}$ & $\begin{array}{r}18 \\
3 \\
1\end{array}$ & $\begin{array}{r}16 \\
3 \\
1\end{array}$ \\
\hline
\end{tabular}

Thirty-two cases had advanced massive fibrosis (Category C-D, Davies and Mann, 1949). Sixteen of them had electrocardiographic signs of pulmonary heart disease. Marked right ventricular hypertrophy was found at necropsy in all 16 cases. In 14 of these, emphysema of the bullous type and of considerable degree was present, but in the other two with the same degree of right heart hypertrophy emphysema was strikingly absent (Fig. 1). These two cases had the same electrocardiographic signs of right ventricular hypertrophy as similar cases in which emphysema was also present.

A further nine cases with similar advanced massive fibrosis had electrocardiographic changes 
which were only suggestive of right cardiac change. Five of these had marked deviation to the right of the electrical axis with prominent pointed $P_{\mathrm{II}}$ and $\mathbf{P}_{\text {III }}$, two others had the pulmonary $\mathbf{P}$ waves only, and two had the pattern of predominant $S$ waves in standard leads I, II, and III. The chest leads did not show the typical signs of right ventricular hypertrophy in these nine cases. Only two hearts of the nine showed marked right ventricular hypertrophy at necropsy. Emphysema was found in six cases. Electrocardiographic change towards the vertical heart pattern with right axis deviation and development of $\mathbf{P}$ pulmonale has been present in cases which have shown early right ventricular hypertrophy at necropsy shortly afterwards, and have had severe massive fibrosis. This phase might warrant the use of the term right ventricular stress as a clinical description.

The remaining seven cases of advanced massive pneumoconiosis had a normal QRS pattern and all but one had normal ST segments. In the exception the ST segment was depressed with flat $T$ waves, thought to indicate ischaemic damage, but the muscle was found to be normal and the right ventricle slightly hypertrophied. Adherence of the pericardium may have been responsible for the ST changes in this case.

There were 17 cases with small masses (Category A-B, Davies and Mann, 1949), and it is noteworthy that only three of these produced the electrocardiographic pattern of pulmonary heart disease and these three were the only ones to show right ventricular hypertrophy at the subsequent post-mortem examination. Four of the other cases had some electrocardiographic change; right axis deviation with pulmonary $\mathbf{P}$ waves in three and right bundle branch block in one.

Seven cases of simple pneumoconiosis were studied. In four of them emphysema and bronchitis were the most marked findings and in a fifth there was carcinoma of the lung. One of the four cases with marked emphysema showed right ventricular hypertrophy in the right chest leads and three had pulmonary $P$ waves. One case with simple pneumoconiosis as the principal finding had pulmonary $\mathbf{P}$ waves in the electrocardiogram. The last case had auricular fibrillation which in our experience is so uncommon in pulmonary heart disease that another cause was suspected although not found. Of the seven cases, one only showed right ventricular hypertrophy at necropsy, and this had been evident clinically.

The Vascular Lesion

Right ventricular hypertrophy occurs in coalworkers' pneumoconiosis. It occurs with greater frequency and is of greater degree in massive fibrosis than in simple pneumoconiosis. In our material early right ventricular hypertrophy detectable by weighing the ventricles was present in $34 \%$ of the massive fibrosis and in $46 \%$ of simple pneumoconiosis. In sharp distinction, marked right ventricular hypertrophy, recognizable in the thickness of the right ventricular wall and often detectable during life in the electrocardiogram, was present in $57 \%$ of those with massive fibrosis but only in $17 \%$ of the cases of simple pneumoconiosis.

In both forms of the disease many cases have other complicating features which may affect the right heart, particularly chronic bronchiolitis and emphysema, but in simple pneumoconiosis even with these marked right ventricular hypertrophy is not frequent. In massive fibrosis cases without these complicating factors produce marked right ventricular hypertrophy. The difference in the incidence of right ventricular hypertrophy suggests that there is a factor operating in massive fibrosis not present in simple pneumoconiosis.

One striking pathological difference between the two disease states is a progressive stenosis of the vessels in the pulmonary arteriolar bed in massive fibrosis (Fig. 2). In and adjacent to the areas of massive fibrosis the pulmonary arterioles showed destruction of the media, invasion of the wall with dust-laden macrophages, and gross intimal thickening with progressive stenosis (Wells, 1954b). The larger the areas of massive fibrosis, the greater the number of vessels involved in the process. This is a slowly progressive stenosing arteriolar lesion and so is capable of producing obstruction to pulmonary blood flow, increased right ventricular work, and eventual right ventricular hypertrophy.

The pulmonary arteriolar stenotic changes are not found in the vessels of the non-fibrosed dust foci of simple pneumoconiosis and right ventricular hypertrophy would not occur from this cause (Fig. 3).

Six cases recorded above with advanced massive pneumoconiosis but without marked right ventricular hypertrophy show a complete absence of the arteriolar lesion in two instances and changes of slight degree in four. This lends support to the association of hypertrophy and the arteriolar lesion although the number is small; it also helps to explain the occasional anomaly of massive fibrosis present for a long period without signs of right cardiac change. We have observed that there may be a latent period, occasionally up to 20 years, when the lung lesions may be detected and measured radiologically without there being clinical evidence of right cardiac change. The reason for 


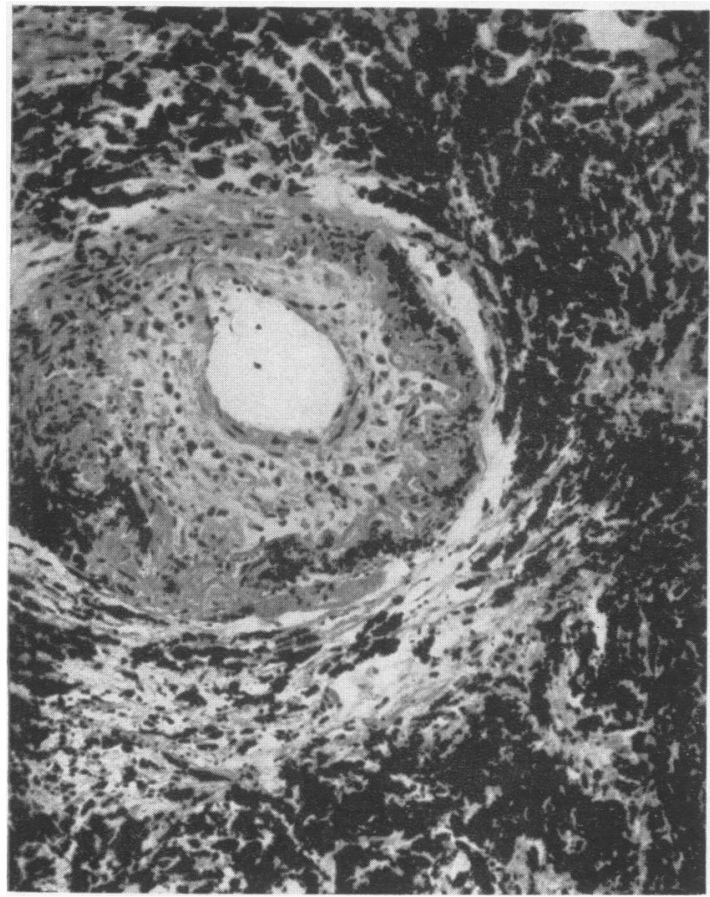

Fig. 2.-The arteriole shown was in a massive dust lesion. The lumen was much reduced by thickening of the vessel. The adventitia and media contained dust-laden macrophages and the structure of the vessel was destroyed. $\times 155$.

the absence of pulmonary arteriolar lesions in occasional cases of massive fibrosis is not known. When the pulmonary arteriolar changes have reached a degree sufficient to cause restriction of pulmonary blood flow on exercise and increased right ventricular work then the process becomes irreversible. It is evident that even then years may elapse during which the right ventricle hypertrophies but does not fail. The right ventricular failure comes when there is great hypertrophy and inability to meet the increasing load. In many cases there are the other complicating features-emphysema, bronchiolitis, tuberculosis, or intercurrent diseasewhich precipitate heart failure earlier or cause anoxic failure or death from other causes. In these cases right heart failure may not be evident and the lesser degree of right ventricular hypertrophy only found if looked for carefully.

The evidence suggests that in simple pneumoconiosis without collagenous fibrosis and without obstructive arteriolar lesions, marked right ventricular hypertrophy is uncommon, and the lesser degrees of hypertrophy which are found are usually in association with chronic bronchiolitis and emphysema.

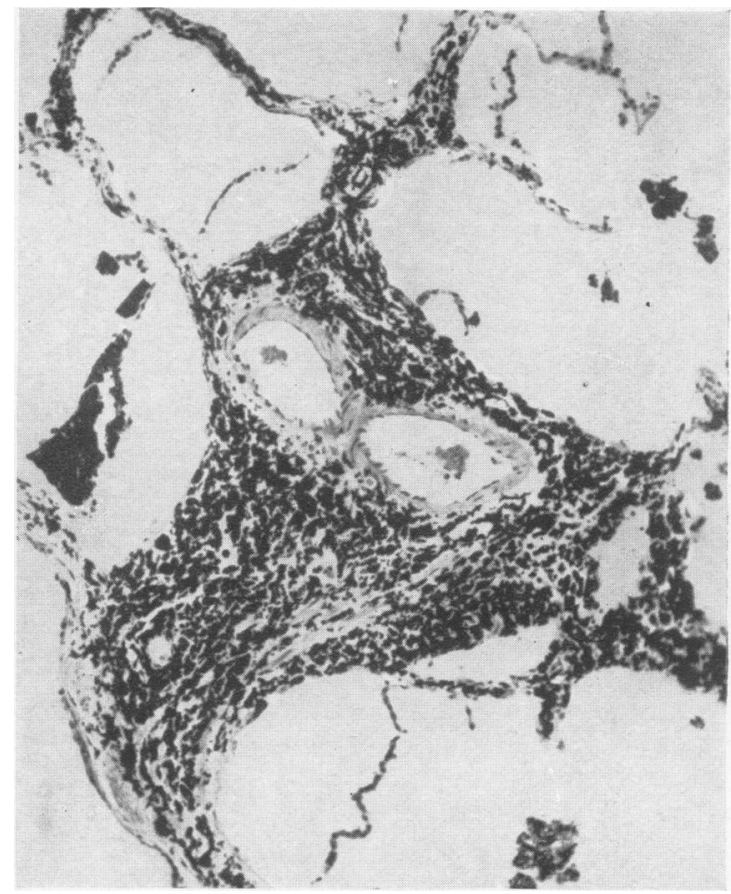

Fig. 3.-The two arterioles shown were in a dust focus from a case of simple pneumoconiosis. The structure of the vessels was normal. $\times 100$.

Electrocardiographic correlation indicates that when right ventricular hypertrophy appears in massive fibrosis it develops to an extent detectable in the electrocardiogram. In simple pneumoconiosis this is not so but many of these cases show a pattern of the vertical heart with tall pointed $\mathbf{P}$ waves in leads II and III. It is suggested that this indicates a stage of right heart stress in the presence of pulmonary disease.

\section{Summary}

Cardiac hypertrophy in coalworkers' pneumoconiosis has been studied. The overall pattern is presented in an analysis of 1,000 necropsies. The degree of right ventricular hypertrophy has been analysed by weighing the ventricles in 80 cases and comparing its extent with the pneumoconiosis in the lungs. In 56 cases in which clinical details have been available the electrocardiogram and the right ventricular hypertrophy at necropsy are compared.

The evidence suggests that right ventricular hypertrophy is frequent and of considerable degree in massive fibrosis but much less common and of a lesser degree in simple pneumoconiosis. Another difference between the two disease states is in 
massive fibrosis a progressive stenosing arteriolar lesion restricting the pulmonary vascular bed, a condition not found in simple pneumoconiosis. This vascular lesion is postulated as the cause of the marked degree of right ventricular hypertrophy in massive fibrosis, and it is shown that this can be so when complicating factors such as emphysema are not present. In simple pneumoconiosis the frequent presence of bronchiolitis and emphysema causes some right cardiac change but not to the same extent.

The electrocardiogram does not identify the early right ventricular hypertrophy although it shows features such as a vertical heart pattern with pulmonary $\mathbf{P}$ waves in these cases. In massive fibrosis the electrocardiogram detects the right ventricular hypertrophy when it reaches a marked degree and before heart failure appears.
We wish to thank Dr. J. C. Gilson, Director of the Pneumoconiosis Research Unit (M.R.C.), Llandough Hospital, and members of his staff for permission to study cases under their care and for their help. We are grateful to Professor J. Gough, Department of Pathology, Welsh National School of Medicine, for his advice and criticism.

\section{REFERENCES}

Davies, I., and Mann, K. J. (1949). Proc. 9th int. conference industr. med. London, 1948, p. 769. Wright, Bristol.

Fletcher, C. M., and Gough, J. (1950). Brit. med. Bull., 7, 42.

Gough, J. (1952). A paper read before the Fourth Conference of the McIntyre Research Foundation on Silicosis, Noranda, Quebec, January 1952

- , and Wentworth, J. E. (1949). J. roy. micr. Soc., 69, 231

Heppleston, A. G. (1953). J. Path. Bact., 66, 235.

Heppleston, A. G. (1953). J. Path. Bact., 66, 235.

Herrmann, G. R., and Wilson, F. N. (1922). Heart,

Lavenne, F. (1951). Rev. belge Path., 21, Supp

Thomas, A. J. (1948). Brit. Heart J., 10, 282.

(1951). Ibid., 13, 1

(1952). Abst. European Congress Cardiology, p. 65.

Wells, A. L. (1954a). Brit. Heart J., 16, 74. 